International Journal of Canadian Studies

Revue internationale d'études canadiennes
INTERNATIONAL JOURNAL OF CANADIAN STUDIES

REVUE INTERNATIONALF D'ÉTUDES CANADIENNES

\title{
Introduction : entre idéal canadien et attraction étatsunienne
}

Godefroy Desrosiers-Lauzon et Serge Dupuis

Numéro 44, 2011

Americanity, Continentalism and Globalisation

Américanité, continentalité et mondialisation

URI : https://id.erudit.org/iderudit/1010077ar

DOI : https://doi.org/10.7202/1010077ar

Aller au sommaire du numéro

Éditeur(s)

Conseil international d'études canadiennes

ISSN

1180-3991 (imprimé)

1923-5291 (numérique)

Découvrir la revue

Citer ce document

Desrosiers-Lauzon, G. \& Dupuis, S. (2011). Introduction : entre idéal canadien et attraction étatsunienne. International Journal of Canadian Studies / Revue internationale d'études canadiennes, (44), 7-22.

https://doi.org/10.7202/1010077ar d'utilisation que vous pouvez consulter en ligne.

https://apropos.erudit.org/fr/usagers/politique-dutilisation/ 


\title{
Godefroy Desrosiers-Lauzon et Serge Dupuis
}

\author{
Introduction : entre idéal canadien \\ et attraction étatsunienne
}

\author{
Pour un dossier spécial sur les Canadiens aux États-Unis
}

\section{Le projet canadien}

Peut-on extraire un sens du Canada ? S'est-il seulement défini par la négative, n'a-t-il représenté qu'une ombre de son voisin puissant ? À la suite de négociations constitutionnelles qui ont accentué les clivages idéologiques dans la deuxième moitié du $20^{\mathrm{e}}$ siècle, plusieurs citoyens du Canada arrivèrent à un constat que leur pays n'avait été qu'un hasard de l'histoire. Nombreux sont ceux qui pensent que les nuances entre Canadiens et Américains sont insignifiantes. Pourtant, une telle conception n'explique en rien l'existence actuelle du Canada et obscurcit ses continuités historiques. L'anti-américanisme se fonde-t-il sur de simples préjugés ou est-il aussi tributaire de la tradition politique britannique et de la préoccupation pour la pérennité d'une culture collective particulière?

Ce travail de repérage des caractéristiques « canadiennes » fut entrepris il y a une décennie par une multitude de chercheurs. Pour l'historienne Nancy Christie, le Canada britannique s'est formé en réaction à la Révolution américaine. À son avis, on sous-estime le second souffle que la consolidation de la colonie canadienne a donné à l'Empire britannique. La création d'institutions britanniques, les interactions avec Londres, la fidélisation à l'Empire par les Églises protestantes et l'arrimage du droit civil et de l'Église des descendants français aux institutions britanniques représentent un important maillon de l'histoire canadienne (Christie, Transatlantic Subjects, 19). Il est bien connu que les historiens Michel Brunet et José Igartua ne s'entendaient pas sur les conséquences de la défaite de la France en 1763, mais ils s'entendaient pour dire que la politisation des Canadiens français et leur conscientisation collective a émergé entre la fin du XVIII et le milieu du XIX ${ }^{e}$ siècle et non pas avant la Conquête (Dumont, Genèse de la société; Brunet, « Les Canadiens après la Conquête "; Igartua, "A Change in Climate"). La création des assemblées législatives des Haut-Canada et Bas-Canada en 1791, tout comme la confirmation du droit à la propriété et l'attribution de la responsabilité gouvernementale en 1849 , plurent à une importante part de la population, ce qui explique, du moins en partie, l'absence de " Révolution canadienne ». Dans la dernière décennie, les historiens canadiens Carol Wilton et Jerry Bannister ont minimisé l'importance des Rébellions de 1837-1838 en estimant que les législateurs nommés par Londres qui prenaient en compte les griefs de leurs sujets avaient formé « an effective precursor to responsible government » (Bannister, The Rule of Admirals), soit le début d'une lente marche vers la responsabilité 
gouvernementale. Malgré tout, ces auteurs pourraient difficilement estimer que l'hostilité de l'armée britannique envers les Patriotes était marginale dans la trajectoire historique du Canada. Cette manifestation de frustrations, particulièrement importante au Bas-Canada, signalait minimalement que la démocratisation avançait trop lentement.

On associe l'historien Ian McKay à une reconfiguration de l'historiographie canadienne qui minimise la Confédération comme moment décisif. Il voit le siècle qui s'est écoulé entre 1840 et 1940 comme celui de la construction d'un État libéral, toujours affilié à la Couronne, mais qui privilégia le développement du capitalisme et la responsabilité gouvernementale (McKay, "The Liberal Order-Framework", 6). La Confédération de 1867, la colonisation de l'Ouest, l'abandon de la National Policy et des tarifs commerciaux préférentiels, l'abandon de l'ultramontanisme par l'Église canadienne-française vers 1900 ainsi que la codification des lois civiles et criminelles représenteraient des victoires pour le libéralisme politique.

Malgré ses nobles objectifs, le projet libéral avait aussi ses revers. Au grand malheur des femmes, des Autochtones, des travailleurs et des catholiques, il entraîna le recul de plusieurs libertés dont jouissaient ces groupes avant sa consolidation. Alors que les Autochtones pratiquaient un égalitarisme social semblable à certains égards à l'individualisme britannique, la création des réserves territoriales priva ces derniers, selon l'historien Robin Jarvis Brownlie, de la propriété, de l'impôt et de la libre circulation (Jarvis, "A Persistant Antagonism", 312). Pour sa part, l'historienne Adele Perry constate que l'implantation d'une élite victorienne en Colombie-Britannique a durci les attentes morales et raciales envers les colons en exigeant l'abandon de leurs liens commerciaux, personnels et amoureux avec les non-Européens (Perry, On the Edge of Empire, 7). De son côté, la juriste Constance Backhouse rappelle que plusieurs des premières lois canadiennes restreignaient ou abolissaient les recours au divorce et à l'avortement. Comme elle le constate : "Sexuality proved to be one of the broadest webs for nineteenth-century legal entanglement », (Backhouse, Petticoats and Prejudice, 327). Selon la démonstration du politologue Benedict Anderson, à la base, la nation se veut une communauté d'appartenance inclusive et un projet politique qui aspirent à la représentativité; on ne peut nier qu'elle a également exclu des pans majeurs de la population qu'elle estimait représenter. Prétextant l'indignité à accéder au statut de citoyen, les États-nations ont souvent assuré la mainmise d'une élite sur la destinée d'une majorité de leurs habitants. Il aura fallu que ces «marges » obtiennent l'accès à l'égalité au terme de luttes épiques pour enfin atteindre un contrat social plus juste.

C'est dire que le projet politique canadien de « liberalism with a crown » offre un cadre d'analyse utile à condition qu'on prenne en compte ses limites et contradictions. Cette culture politique de démocratie passive semi-royaliste 
constitue donc une première caractéristique particulière du Canada. L'attitude et la relation aux cultures coloniales et autochtones forment un deuxième particularisme du Canada par rapport aux États-Unis. Jocelyn Létourneau y voit une « canadianité » qui représente, à son sens, une volonté entre Anglophones, Francophones, Autochtones et autres groupes ethniques de vivre ensemble dans un État bilingue (Létourneau, Passer à l'avenir, 89). Létourneau entretient néanmoins des réserves sur la véritable réussite de ce modèle qui intègre trop peu ses « minorités » à la politique fédérale, au risque de l'engloutissement des non-anglophones. La promotion de la diversité, croit-il, ne reflète pas suffisamment les raisons communes des Francophones et des Autochtones qui ont vécu leur aventure de manière distincte.

Un autre facteur explique l'affinité des Canadiens pour la pérennité culturelle : la simple prédominance des citoyens d'origines française et anglaise. C'est Howard Palmer qui démontre que le Canada se distinguait des États-Unis sur le plan ethnique. Il a relevé que jusqu'en 1920, $92 \%$ de la population était d'origine française ou anglaise, malgré l'immigration du tournant du siècle. Alors qu'aux États-Unis, 71 \% des immigrants provenaient de l'Europe centrale, du Sud ou de l'Est, la proportion équivalente s'élevait à seulement un tiers au Canada en raison de l'importance du contingent britannique (Palmer, “Mosaic vs. Melting Pot?", 491-3). La proportion de catholiques a longtemps frôlé les 50 \% au Canada alors que ces derniers étaient bien moins présents aux États-Unis $(I d, 499)$. Palmer explique donc la politique de multiculturalisme au Canada comme une prise en compte des origines diverses du pays, mais aussi comme un moyen de résister au principe du biculturalisme qui tient à cœur aux Canadiens français. Au début des années 1970, les Britanniques (15\%), Allemands $(13 \%)$ et d'autres groupes à proportions semblables, formaient les origines les plus communes aux États-Unis. Par contre, au Canada, les Britanniques (45 \%), les Français (29 \%) et une myriade d'autres groupes formaient la «mosaïque canadienne » (524-5). L'identité et l'idéologie politicoculturelles du pays posséderaient donc aussi un fond substantiel.

L'essayiste John Ralston Saul va plus loin dans son analyse du tronc culturaliste du Canada. Selon lui, la culture distincte de ce pays est principalement le résultat d'un métissage de cultures européennes et autochtones. C'est ainsi qu'on arriverait à expliquer la nature «métisse » ou « égalitariste » des Canadiens qui la distingue de celle de leurs voisins. N'était-ce pas Samuel de Champlain qui clamait « nos fils marieront vos filles et nous deviendrons un peuple » dès les premiers jours de la colonie ? Selon l'auteur, l'attitude environnementaliste, nomade, militaire et diplomatique des Autochtones expliquerait le pacifisme des Canadiens et la préoccupation générale des cours de justice de minimiser les torts commis aux minorités (Saul, A Fair Country, 74). Saul avance à juste titre que les cultures ne sont pas statiques et qu'elles s'influencent mutuellement, ce qui expliquerait la relation particulière qu'entretiennent les Canadiens avec la culture. Malheureusement, sa lecture 
anhistorique et psychanalyste de plusieurs documents, ainsi que sa négligence à intégrer les effets de la laïcisation et des piètres ressources investies par le Royaume-Uni pour consolider un État centralisé, affaiblit sa bien noble intention de placer la préoccupation « égalitariste » au centre du projet canadien.

\section{Le projet canadien-français}

Qu'il ait été un projet national généreux, insuffisant ou discriminant, le Canada comme nation n'a pas fait consensus, malgré ses efforts rassembleurs. Bien qu'une culture populaire " canadienne » ait existé en Nouvelle-France et au Bas-Canada, la consolidation politique d'une conscience collective canadiennefrançaise ne s'est pas réalisée avant l'échec des Rébellions. Les Canadiens français furent largement en accord avec les réformes politiques du milieu du $\mathrm{XIX}^{\mathrm{e}}$ siècle, mais l'écrasement des espoirs républicains permit l'émergence de l'Église comme institution para-étatique et hégémonique d'expression de la collectivité française d'Amérique.

Comme toute consolidation de projets impériaux se base sur la fusion de la colonie à la métropole, le projet canado-britannique justifia une volonté croissante d'assimiler ses citoyens à la culture britannique. En reconnaissance du soutien de l'Église pour écraser les Rébellions, Londres permit la restitution en 1839 des propriétés jésuites (confisquées en 1763) à l'Église, ainsi que l'installation de congrégations religieuses (Oblats, Jésuites, Sœurs Grises, etc.) expulsées de France dans la seconde moitié du XIX ${ }^{e}$ siècle. Par contre, si l'Église prit la place des Républicains (parfois par le ridicule et l'excommunication), elle ne s'est pas mise à promouvoir l'impérialisme canado-britannique, mais plutôt à tolérer son emprise sur le politique en échange de la liberté de développer une culture intellectuelle, artistique et historique proprement canadienne-française. Elle tâcha donc de construire une référence culturelle et des représentations fortement inspirées du Vatican et de la France, mais aussi de mettre sur pied nombre d'institutions modernes comme les écoles, les hôpitaux et les orphelinats. L'État, pour sa part, s'engageait modestement dans ces sphères jusqu'au mitan du $\mathrm{XX}^{\mathrm{e}}$ siècle. La fin du $\mathrm{XIX}^{\mathrm{e}}$ siècle fut particulièrement éprouvante pour la jeune nation : la hausse de la natalité au Québec (bien encouragée par l'Église qui voulait contrecarrer les visées assimilatrices de Lord Durham) occasionna un surpeuplement des terres laurentiennes, alors que la concurrence agricole de l'Ontario et de l'Ouest canadien mena le tiers de sa population à s'exiler de la mère patrie. Au tournant du $\mathrm{XX}^{\mathrm{e}}$ siècle, $10 \%$ de la population de Nouvelle-Angleterre était canadienne-française; elle devint majoritaire dans le Madawaska au Nouveau-Brunswick, dans nombre de cantons de l'Est, et dans le Nord ontarien (Frenette, Brève histoire, 84-6). L'arrivée de ces migrants provoqua un contrecoup identitaire de la part des Anglophones natifs de ces régions, qui se mirent à interdire l'instruction en français dans toutes les provinces canadiennes. Alors qu'on avait toléré la construction nationale du Canada français pendant la majeure partie du $\mathrm{XIX}^{\mathrm{e}}$ 
siècle, les Canadiens anglais devinrent de plus en plus intolérants en regard de cette langue distincte au tournant du $\mathrm{XX}^{\mathrm{e}}$ siècle. Cette atteinte grandissante des libertés des Canadiens français contribua également à renforcer la nation comme référence para-étatique.

Le refus d'accorder aux Canadiens français des droits scolaires équivalents à ceux de la minorité anglo-protestante du Québec suscita un mécontentement palpable (Silver, The French-Canadian Idea). Le politicien Henri Bourassa et d'autres intellectuels vinrent à définir le Canada comme un pacte qui assurerait le respect des droits des deux «peuples fondateurs » (Lacombe, La rencontre de deux peuples). L'élite canadienne-française du Québec se porta à la défense des minorités francophones en instituant des organismes de solidarité, comme les Associations catholiques de la jeunesse canadienne-française (1903) et le Conseil de la vie française en Amérique (1937), qui firent la promotion du développement institutionnel des communautés canadiennes-françaises hors Québec (Martel, Le deuil d'un pays imaginé; Gervais, Des gens de résolution). Pour contrecarrer la marginalité du français sur la place publique, le CVFA lança des campagnes de souscription qui permit de lancer et de soutenir le quotidien L'Évangéline en Acadie et des radios françaises dans l'Ouest (Martel, Le deuil, 44).

L'après-guerre fut toutefois le théâtre de mutations sociopolitiques importantes. Les Canadiens français du Québec, frustrés par leur marginalisation sociale et économique, vinrent à exiger l'intervention de l'État provincial dans la poursuite de leur épanouissement national. L'effet de la Révolution tranquille fut doux et amer pour les Canadiens français à l'extérieur de la Belle Province : ils réussirent à décrocher des concessions linguistiques en matière d'instruction et de services provinciaux, mais ils perdirent également leur place fondamentale dans la représentation collective du Canada français et furent exclus des nouvelles aspirations québécoises (Belliveau, “Acadie's Ambivalent Leap into the Liberal Order"). C'est ainsi que plusieurs institutions nationales, comme l'Ordre de Jacques Cartier et les Sociétés Saint-Jean-Baptiste se désagrégèrent peu à peu. L'éclatement du consensus national canadien-français reflétait également le ralentissement du flux migratoire vers l'extérieur de la province. Alors que $8 \%$ des Québécois vivaient à l'extérieur de leur province en 1921, cette proportion ne s'élevait plus qu'à $3 \%$ en 1971 (Frenette, Brève histoire, 172). Pour leur part, les minorités ne s'en sont jamais vraiment remis...

Les écrits récents sur le Québec du $\mathrm{XX}^{\mathrm{e}}$ siècle interprètent moins la Révolution tranquille comme un rejet politique de l'omniprésence de l'Église et davantage comme une transformation culturelle qui, appuyée par des éléments du clergé, aurait mené à la laïcisation et l'Étatisation du Canada français par l'entremise du gouvernement du Québec. Par exemple, le mouvement personnaliste de l'Église française fit la promotion de l'authenticité personnelle et de l'action sociale auprès de son prochain, chez les jeunes des années 1930 et 1940. 
La pléthore d'organismes et d'institutions créés pour répondre au dépaysement causé par l'industrialisation et l'urbanisation occasionna une reprise en force de l'Église jusqu'aux années 1960. L'historien Michael Gauvreau comprend ce désir d'institutionnaliser la gestion des conflits sociaux et de maintenir la famille comme unité sociale de base, comme le moyen d'assurer la primauté des valeurs chrétiennes dans un contexte qui, autrement, aurait entraîné son déclin (Gauvreau, Les origines catholiques, 59). Dans la majorité des cas, l'État prit la relève des institutions confessionnelles avec souplesse. Malgré les espoirs des personnalistes, les pratiques américaines de fréquentation et de divertissement gagnèrent ainsi du terrain après 1960. La religion fut alors reléguée à la sphère privée et la société perdit une grande part de sa cohésion sociale, selon Gauvreau, puisque l'État ne put projeter son appareil dans toutes les sphères de la société, d'une façon qui aurait pu pallier à l'absence de l'ancienne Église nationale (Id, 304).

Environ un demi-siècle après la Révolution tranquille (et la révolution encore plus tranquille qui éloigna les Canadiens anglais des références britanniques durant les années 1950 et 1960), le Canada, le Québec et le Canada français demeurent des projets inachevés. Le Canada vit toujours dans l'ombre de son puissant voisin, alors que le Québec et le Canada français peinent à colmater la brèche de l'assimilation anglophone à l'extérieur du Québec. D'un côté, Ottawa offre peu de moyens pour assurer cette pérennité; et de l'autre, Québec n'arrive pas à convaincre sa population de la viabilité du projet souverainiste. Certains diront que le Canada se trouve dans une impasse, alors que d'autres feront remarquer que le quasi consensus sur les accommodements culturels et la tradition politique britannique demeurent des remparts importants pour éviter l'enfouissement total dans la culture et l'économie américaines.

\section{Le rêve américain et la migration canadienne vers le Sud}

Nous avons précédemment remarqué que le projet politique canadien n'avait pas empêché la migration vers la République américaine. En fait, il serait plus juste de dire que la plupart des émigrants sont partis sans que le projet national intervienne dans leur décision. Déçus par une pénurie de travail ou une philosophie politique jugée trop traditionaliste, plusieurs gens ordinaires « votèrent avec leurs pieds » en cherchant leur bien de l'autre côté de la frontière, pas nécessairement en opposition à la nation, mais d'une façon qui tendait à nier sa pertinence, où à forcer sa remise en question.

Ainsi, l'historien Jacques Rouillard a remarqué que les femmes ayant migré aux Petits Canadas au début du $\mathrm{XX}^{\mathrm{e}}$ siècle définirent leur parcours comme une libération du fardeau domestique en milieu rural, comme une entrée longtemps désirée dans l'orbite du salariat et de l'économie monétaire Par ailleurs, la migration massive des Canadiens français vers la Nouvelle-Angleterre entre 1840 et 1930 força une remise en question de l'idée de nation entretenue 
par les élites. L'historien Yves Roby a démontré que les élites canadiennesfrançaises, après un moment d'incompréhension, en sont venues à renforcer les projets de colonisation rurale dans le Nord québécois et ontarien, puis à redéfinir les migrants comme des missionnaires qui étendraient les frontières du Canada français tout en promouvant l'épanouissement du catholicisme aux États-Unis. En définissant la nation ainsi, on évoquait la symbolique de l'Amérique française d'avant 1763.

Les métarécits nationaux canadiens, francophones et anglophones furent remis en question suivant la relation entre le Canada et les États-Unis en général, et les mouvements migratoires des Canadiens en particulier. Cette reformulation récursive de la culture, de l'idéologie et des institutions, par l'expérience et l'action cumulatives de leurs humbles sujets, se retrouve dans les écrits de Clifford Geertz et Henri Lefebvre.

Les migrations semblent ainsi échapper à l'influence des projets politiques. Une partie de l'explication se trouve, depuis la fin du XIX ${ }^{\mathrm{e}}$ siècle, dans l'imposant corpus de l'étude des migrations. Selon Ernest G. Ravenstein, les migrations sont généralement le fruit de facteurs économiques. Leur volume et leur qualité seraient fonction des différences entre le marché du travail de la destination des migrants, et celui de leur patrie. Ravenstein propose aussi l'idée que les migrations seraient facilitées par la proximité géographique. Les migrations seraient aussi ponctuées d'étapes, c'est-à-dire que le migrant « arrive à destination » en un endroit qui n'était pas toujours celui planifié au départ, à la suite d'une série de déplacements qui le ramènent, parfois, à son lieu d'origine. Enfin, la modernisation économique (dont l'économie monétaire et le consumérisme), l'urbanisation, et l'amélioration des moyens de communication et de transport qui l'accompagnent, encourageraient les migrations (Cadwallader, Migration and Residential Mobility, 39).

Ce modèle fut raffiné, notamment dans les années soixante par Everett Lee, qui proposa d'analyser les facteurs économiques qui incitent l'émigration et qui les attirent vers certaines destinations (Id 9-10, 39-40) Cette analyse des push et pull factors a inspiré plusieurs recherches, parce qu'elle est compatible avec les présupposés individualistes et rationalistes de l'idéologie républicaine et de la théorie économique néoclassique (Moon, « Paradigms in Migration Research », 507). Lee porta attention aux « obstacles » qui préviennent, ralentissent, compromettent, et parfois transforment le parcours migratoire en cours de route. Parmi ces obstacles se trouvent les frontières politiques - celles-ci, fort poreuses en Amérique du Nord, ne furent pas une entrave majeure aux déplacements. Pour Lee, il importe aussi de moduler les facteurs économiques de la migration selon la réponse différenciée des individus à ces facteurs selon leur sexe, leur âge, leur culture, leur classe sociale et selon les événements importants de leur cycle de vie, ainsi qu'en fonction de leur niveau d'éducation et d'accès à l'information. 
Ce modèle, somme toute économétrique (et comportementaliste), s'applique fort bien au contexte américain, où l'intensité et l'étendue des réseaux économiques ont facilité les flux migratoires. Le contexte de modernisation et d'urbanisation rapide en Occident depuis le XVIII siècle a structuré les migrations d'Amérique et celles-ci forment un élément fondamental de la composition et de la structure des sociétés qui en sont issues (Bodnar, The Transplanted, 5-30). C'est ce contexte qui facilita le mouvement des Canadiens français vers la Nouvelle-Angleterre, puis ailleurs aux États-Unis lorsque les voies de communication le permirent. On constate alors que les Canadiens et les Étatsuniens font preuve, depuis longtemps, d'une grande mobilité géographique, bien que celle-ci ne se soit pas toujours traduite en termes de mobilité sociale (Thernstrom, Poverty and Progress, 85-90, 102-104).

Ce modèle pétri d'individualisme méthodologique n'a pu occulter d'autres facteurs, historiques, sociaux, ou culturels. Les migrations du passé influencent celles à venir : les migrants iront là où d'autres les ont précédés. Ils mobiliseront leurs réseaux sociaux (famille, parenté, amis, voisinage) afin de faciliter leur collecte de renseignements, leur déplacement et leur implantation. La taille de la communauté expatriée, soit le nombre de migrants d'une origine donnée concentrés dans une destination donnée, sera un facteur facilitant. Ainsi les migrants se déplaceront en circuits (c'est-à-dire le long de voies, entre les lieux de départ, d'arrivée et de retour) et en chaînes (c'est-à-dire grâce à l'appui et à l'encouragement des proches et des « intermédiaires ethniques » qui les auront précédés), en flux et contre-flux, entre des pôles où ils tendent à se regrouper (Woods, « Migration Theories »).

L'école d'analyse économétrique des migrations a trouvé un terreau fertile dans le contexte canadien. Or, modernisation économique, urbanisation et migration y sont généralement interprétées par l'intelligentsia comme des facteurs de convergence avec les États-Unis, voire des facteurs d'intégration économique et culturelle. Dès le débat des années 1870 sur le protectionnisme tarifaire (cf. Woodsworth, dans ce dossier spécial), cet amalgame entre modernisation et influence économique étatsunienne structure le rapport des élites canadiennes aux États-Unis. Au terme du $\mathrm{XX}^{\mathrm{e}}$ siècle, le journaliste Jeffrey Simpson se veut rassurant et conclut que les Canadiens ressemblent de plus en plus aux Américains, sans devenir américains. Son constat n'est cependant pas appuyé par une démonstration substantielle de cette différence. Selon l'historien Reginald C. Stuart, l'intensité de la relation entre les deux voisins ne menacerait pas l'existence du Canada, mais serait fondée sur des similitudes culturelles, et représenterait une nécessité économique pour la plupart des Canadiens. Reprenant le point de vue de l'école Hansen-Brebner des relations Canado-américaines (Buckner, « How Historians Stopped Worrying », 118-9) en se plaçant ostensiblement du point de vue des parties prenantes (stakeholders) de cette relation, Stuart démontre la construction historique de 
cette relation, en rapport avec l'action incrémentielle de générations d'individus et la fréquentation mutuelle de leurs communautés d'intérêts.

Comme le démontre Simpson, l'après-guerre fut marquée par une forte émigration de Canadiens scolarisés en direction des États-Unis, résultat d'une différence marquée entre les salaires moyens des deux nations en contexte de croissance économique rapide. L'inquiétude sur la pérennité de la nation canadienne atteignit alors un sommet, y compris pour les historiens nationalistes Donald Creighton et W.L. Morton, alors que les élites nationales s'alarmèrent de l'intégration économique entre les deux voisins et de l'apparente impuissance des gouvernements à résister à cette évolution. Pour l'occasion, on emprunta à la Grande-Bretagne le terme «brain drain », que l'on traduisit par l'exode des cerveaux. L'entrée en vigueur du Preference System comme régime d'immigration en 1965, en favorisant la réunification familiale au détriment de l'immigration en provenance de nations occidentales (dont le Canada), combinée à la croissance des tensions sociales aux États-Unis, fit chuter l'émigration dès le milieu des années soixante. Le phénomène a repris une certaine ampleur durant les années 1990, résultat de l'entrée en vigueur du libre-échange avant tout.

Durant les années 1980, la droite canadienne a mis l'exode des cerveaux en épingle (par ex. Basham, « Following the Well-Worn Path South », 15), y voyant une preuve de la supériorité du modèle étatsunien de laisser-faire économique, alors que la gauche et les éléments nationalistes du Parti libéral se sont empressés de nier l'importance du phénomène, de souligner à gros traits la supériorité des politiques sociales et diplomatiques du Canada et de préconiser la diversification des relations économiques étrangères. Par cette récupération idéologique, la droite, tant anglophone que francophone, s'est déplacée de l'anti-américanisme vers une posture plus favorable au modèle américain.

Depuis les années 1990, le corpus de l'étude des régions limitrophes (borderlands) porte une attention particulière aux migrations comme facteur structurant des relations entre les pays d'Amérique du Nord, principalement entre Mexique et États-Unis. Le géographe Randy Widdis, rare spécialiste de la migration des Canadiens anglophones aux États-Unis, constate que la force (et les excès) de l'école des borderlands tient à son attention à la proximité géographique, aux mouvements migratoires et aux facteurs économiques. Selon cette approche, ces trois (vastes) facteurs participent à l'intégration des régions frontalières, des deux côtés des frontières internationales. D'une façon similaire, les géographes Dean Louder et Eric Waddell, négligeant les frontières politiques, ont identifié des zones concentriques de dispersion de l' « archipel » francophone d'Amérique du Nord, autour du centre laurentien. De facto, les Petits Canadas de Nouvelle-Angleterre, ainsi que l'Acadie et l'Ontario français, s'y voient redéfinis comme la «ceinture » de la francophonie 
d'Amérique. Voulant proposer un nouveau modèle de l'Amérique française, ils ont finalement défini la nature du fait français en divers endroits, dont les lieux où le français est un fait de vie publique, où il est un fait de mémoire et où il est un fait d'histoire. Bien que Louder et Waddell ont intégré les Cajuns de la Louisiane dans leur schéma, ils y ont aussi ajouté d'autres endroits phares de la vie française, comme Haïti - un des seuls lieux où l'apprentissage du français est en croissance - et les pôles d'attraction de travailleurs et de retraités francophones comme la Californie et la Floride.

C'est à un Américain ayant immigré au Canada que nous devons une des meilleures analyses des migrations canado-américaines. Robert Harney explique ainsi la migration des Canadiens en Floride : d'abord, des migrants pionniers explorent une destination. Ceux-ci sont souvent des migrants expérimentés, conformément au modèle de Ravenstein, car les pionniers canadiens avaient généralement séjourné ailleurs aux États-Unis, notamment en NouvelleAngleterre, au préalable. Les pionniers rapportent l'information sur la nouvelle destination à leur patrie ou à leur point de départ et une « fièvre » migratoire s'y développe. Les migrants mettront à contribution leurs réseaux sociaux afin de faciliter leur collecte d'information et leur déplacement. Les migrants à destination des Petits Canadas ont ainsi mis à contribution leurs réseaux familiaux et de voisinage. Ce fut aussi le cas en Floride, et ce, pour tous les immigrants, qu'ils soient Canadiens, Étatsuniens, Antillais ou d'autres appartenances ethniques. À destination, des entrepreneurs se spécialisent dans les services aux migrants. Dans le cas floridien, ce sont les premières générations de migrants canadiens en Floride qui ont, depuis les années 1950, offert des services aux migrants et touristes qui les ont suivis. Ces mêmes entrepreneurs ont formé la base de la communauté que les géographes ont appelé Floribec tel que démontré par Louis Dupont et plus tard par Rémy Tremblay (voir aussi le texte de Gilbert et Tremblay dans ce dossier). À terme, ces « courtiers en migration» forment une « intelligentsia organique » (c'est-à-dire structurellement dépendante de la communauté immigrante) et produisent un discours sur la migration.

Cependant, le discours des « courtiers » n'est pas la seule voix parlant de migration. Partout où l'on attribue une importation à l'émigration, un contrediscours apparaît dans la communauté d'origine des migrants, dénonçant la « saignée » qui sape la patrie démographiquement. Les émigrants canadiens à destination des États-Unis ont ainsi fait l'objet d'un contre-discours, c'est le cas des Canadiens français avant 1880, des émigrants qualifiés de l'après-guerre et des années 1990, ou des snowbirds (Coates, Healy et Morrisson, 433, 450). Ce contre-discours est perçu où il y eut une forte émigration, vers une ou quelques destinations précises. Le discours italien à l'endroit des États-Unis durant les années 1920 vient notamment à l'esprit (Gabaccia, Italy's Many Diasporas, 1998, 86-7). 
Le cumul des effets de la migration indique les limites des projets nationaux canadiens, du moins dans leur sensibilité aux forces qui animent le changement social et historique. L'importance de la migration démontre aussi, une fois de plus, le rôle des Canadiens marginalisés dans la (re)composition des projets collectifs. L'expérience et le réseautage des migrants, l'ouverture de canaux de communication entre patries et communautés de la diaspora, l'assimilation de plusieurs migrants, le contre-discours par rapport à l'expérience migratoire finissent tous par influencer lourdement la nation. D'ailleurs, Bruno Ramirez, Donna Gabaccia et Yvan Lamonde, proposent déjà ce constat.

\section{À la fine pointe des recherches sur la tension entre idéal et attraction}

Nous pouvons diviser les textes de ce dossier spécial en " manières d'écrire et de décrire » l'expérience canadienne aux États-Unis. Elles s'identifient à diverses disciplines académiques et à diverses expériences vécues. Nous y retrouvons des récits, une analyse littéraire, un texte géographique, et des travaux d'histoire.

Il sera d'abord question des récits, biographiques et fictifs, de l'expérience au sud des Grands Lacs, des " manières de raconter " l'expérience des Canadiens aux États-Unis. Robert B. Perreault partage sa propre expérience du Petit Canada de Manchester au New Hampshire, parle du point de vue mémoriel d'une part, en relevant les efforts de préservation du patrimoine franco-américain, et d'autre part du niveau scientifique attaché aux disciplines de l'histoire et des études littéraires. Cet engagement de l'auteur avec son objet rappelle le poids croissant de la "public history » et la contribution de la biographie, la mémoire collective et la muséologie à la discipline historique.

En racontant la redécouverte personnelle de son héritage francophone, Caroline LeBlanc, de Watertown (New York), reconstruit symboliquement la nation dont elle est issue, par la voie du récit poétique et biographique. Suivant les précédents des études postcoloniales et ceux de la psychothérapie qu'elle a longtemps pratiquée, l'auteure nous invite à explorer la tension entre les collectivités et l'individu, en portant attention aux non-dits et aux secrets de famille. LeBlanc argumente que l'idéologie de la survivance, croisée avec la proximité géographique entre les Petits Canadas et la mère patrie, ont contribué à maintenir la plupart des Franco-Américains dans les limbes, entre l'intégration et l'isolement. Son texte constate également une marginalité persistante des Franco-américains dans l'imaginaire de la Nouvelle-Angleterre et des ÉtatsUnis. La démarche de l'auteure témoigne aussi des aspirations de cette fameuse troisième génération, celle qui tente de reconstruire l'appartenance ethnique qui a été refoulée par la seconde génération de migrants.

La spécialiste des lettres anglaises Aya L. Gaddas développe une analyse de la communauté imaginée des migrants. En étudiant les personnages franco-américains dépeints par le romancier David Plante, l'auteure constate 
la pénétration des structures géographiques (comme la frontière) et culturelles (comme la religion catholique et la mère patrie) dans les univers mentaux des migrants. Gaddas prend soin de restituer la diversité des interprétations de ces structures, une fois qu'elles sont appropriées par des individus s'intégrant à Providence au Rhode Island.

Ce dossier comporte un texte de la discipline géographique. Pour Anne Gilbert, André Langlois et Rémy Tremblay il s'agit d'un retour à Floribec, dans la communauté francophone implantée autour du comté de Broward en Floride, depuis les travaux pionniers de Louis Dupont, Dean Louder, Anne Gilbert et Rémy Tremblay. Leurs observations sur le terrain, et leur approche issue de la géographie humaine, leur permettent d'interpréter la mobilité saisonnière des hivernants comme la recherche d'un style de vie, qui est bien plus que le lifestyle du commerce. Les Floribécois se concentrent dans un espace communautaire définissable en termes de géographie, d'offre de services et de loisirs à une clientèle francophone, de réseaux de voisinage, d'amitié et de parenté qui s'étendent du comté de la Gold Coast au Canada francophone. Ces réseaux, cette sociabilité en français expliquent la vivacité de Floribec après les sommets de popularité des années 1990s, après que les centres de la communauté dans les commerces et hauts lieux de Sunny Isles et Hollywood aient perdu de leur importance.

La dernière section regroupe des textes issus de la discipline historique. L'historienne Adeline Vasquez-Parra analyse la présence au Massachusetts des Acadiens déracinés par le Grand-Dérangement de 1755. Pour la société d'accueil, c'est une injection d'altérité qui est interprétée en fonction des nombreux bouleversements que vit la colonie au milieu du XVIII ${ }^{\mathrm{e}}$ siècle. Pour les exilés, il est d'abord nécessaire de restaurer les fragments de solidarité qui leur restent. C'est pour leurs familles, foyers de communauté et signes de leur différence, qu'ils concertent leur prise de parole face à une société hôte. Les Acadiens exilés, dès leur premières années d'errance, initient une conversation collective, et avec leurs hôtes, sur leur identité.

Le parcours de la famille Jobin au Massachusetts sur quatre générations depuis 1890 échappe aux explications simples. L'historienne Leslie Choquette dépeint le portrait d'une famille immigrante qui s'approprie de façons fort diverses les modalités d'intégration à la société d'accueil : l'insertion économique et culturelle ainsi que les rapports entretenus (ou pas) avec la mère patrie.

La démonstration de l'historien Jack Little emprunte une méthode similaire à celle de Choquette : par le récit de l'expérience migratoire d'Angus MacKay du comté de Compton jusqu'à Seattle, l'auteur nous convie au déroulement d'un processus biographique informé par une démarche heuristique inspirée de l'histoire sociale. Dans la biographie de MacKay comme dans celle d'innombrables migrants, la mémoire, les réseaux familiaux et le rapport avec 
l'étranger dominent. Par ailleurs, l'expérience de MacKay conduit à la nostalgie d'un passé révolu (le passé de ses ancêtres écossais, et le passé personnel de MacKay dans les Cantons de l'Est), sorte d'écho à la renaissance ethnique faisant vibrer l'Amérique depuis belle lurette.

Par l'entremise du récit des activités et débats qui animèrent les clubs canadiens de New York, l'historien Michael Woodsworth analyse l'impact sur l'identité et la conception même du Canada, qu'eurent les débats sur l'intégration économique entre Canada et États-Unis au tournant du XX $\mathrm{XX}^{\mathrm{e}}$ siècle. Les Canadiens aisés réunis autour des clubs canadiens de New York jouèrent (ou prétendirent jouer) un rôle important dans la mobilisation en faveur d'une intégration commerciale des deux nations. Cela étant posé, l'auteur démontre la complexité de la posture des États-Unis à l'égard du Canada, résultant d'événements, de rapports de classe et d'éléments culturels qui influèrent durablement sur les acteurs historiques.

Ces textes espèrent jeter la lumière sur diverses expériences de migration et d'altérité afin de mieux comprendre les relations et rendre justice aux mythes et réalités qui entourent la relation canado-américaine. Une telle finesse d'analyse n'est pas uniquement souhaitée pour des motifs scientifiques, mais aussi par une quête des enjeux qui lient et opposent les gens, les structures et les cultures. C'est à cela, croyons-nous, que l'expérience des Canadiens aux États-Unis nous convie.

\section{Bibliographie}

Anderson, Benedict. Imagined Communities: A Reflection on the Origins and Spread of Nationalism. London : Verso, 1991.

Backhouse, Constance. Petitions and Prejudice: Law and Women in 19th Century Canada. Toronto : Osgoode Society, 1990.

Bannister, Jerry. "Canada as Counter-Revolution: The Loyalist Order Framework in Canadian History, 1750-1840", Éd. Jean-François Constant et Michel Ducharme, Liberalism and Hegemony: Debating the Canadian Liberal Revolution. Toronto : University of Toronto Press, 2009.

Bannister, Jerry. The Rule of Admirals. Law, Custom and Naval Government in Newfoundland, 1699-1832. Toronto : University of Toronto Press, 2003.

Basham, Patrick. "Following the Well-Worn Path South", Fraser Forum (juillet 2002).

Bélanger, Damien-Claude. «L'antiaméricanisme et l'antimodernisme dans le discours de la droite intellectuelle du Canada, 1891-1945 », Revue d'histoire de l'Amérique française 61.3-4 (été-automne 2008).

Belliveau, Joel. “Acadie's Ambivalent Leap into the Liberal Order”. Éd. Magda Fahrni et Robert Rutherdale, Creating Post-War Canada, 1945-75. Vancouver : University of British Columbia Press, 2008.

Bodnar, John. The Transplanted: A History of Immigrants in Urban America. Bloomington : Indiana University Press, 1985. 
Brownlie, Robin Jarvis. "A Persistent Antagonism: First Nations and the Liberal Order”, Éd. Jean-François Constant et Michel Ducharme. Liberalism and Hegemony. Debating the Canadian Liberal Revolution. Toronto : University of Toronto Press, 2009.

Brunet, Michel. «Les Canadiens après la Conquête : les débuts de la résistance passive ", Revue d'histoire de l'Amérique française 12.2 (printemps 1958).

Buckner, Phillip. « How Canadian Historians Stopped Worrying and Learned to Love the Americans! », Acadiensis 25.2 (printemps 1996).

Cadwallader, Martin. Migration and Residential Mobility: Macro and Micro Approaches. Madison : University of Wisconsin Press, 1992.

Christie, Nancy. Transatlantic Subjects: Ideas, Institutions, and Social Experience in Post-Revolutionary British North America. Montréal : McGill-Queen's University Press, 2008.

Coates, Ken S., Robert Healy et William R. Morrison. "Tracking the Snowbirds: Seasonal Migration from Canada to the U.S.A. and Mexico", American Review of Canadian Studies 32.3 (automne 2002).

Comeau, Robert, et Bernard Dionne. À propos de l'histoire nationale. Sillery : Septentrion, 1998.

Dumont, Micheline. « L'histoire nationale peut-elle intégrer la réflexion féministe sur l'histoire? », À Propos d'histoire nationale, Éd. Robert Comeau et Micheline Dionne, Sillery : Septention, 1998.

Dumont, Fernand. Genèse de la société québécoise. Montréal : Boréal, 1996.

Dupont, Louis, Anne Gilbert et Dean R. Louder. Les Floribécois dans le contexte de la Floride du Sud. Sainte-Foy : Département de géographie, Université Laval, 1994. www.cefan.ulaval.ca/franco/my_html/florida.html, (consulté le 7 juin 2011).

Frenette, Yves. Brève histoire des Canadiens français. Montréal : Boréal, 1998.

Gabaccia, Donna R. Italy's Many Diasporas. Seattle : University of Washington Press, 2000.

Gabaccia, Donna R. "Italian History and gli italiani nel mondo, Part II", Journal of Modern Italian Studies 3 (printemps 1998).

Gauvreau, Michael. Les origines catholiques de la Révolution tranquille. Montréal : Fides, 2008.

Gervais, Gaétan. Des gens de résolution. Le passage du « Canada français » à l'Ontario français. Sudbury : Prise de parole, 2003.

Harney, Robert. "The Palmetto and the Maple Leaf: Patterns of Canadian Migration to Florida", in Shades of the Sunbelt: Essays on Ethnicity, Race, and the Urban South, Éd. R. M. Miller et G. E. Pozzetta. Boca Raton : Florida Atlantic University Press, 1989.

Hansen, Marcus Lee et John Bartlet Brebner. The Mingling of the Canadian and American Peoples. New Haven : Yale University Press, 1940.

Igartua, José. "A Change in Climate: The Conquest and the Marchands of Montreal”, Historical Papers/Communications historiques 9.1 (1974).

Igartua, José. The Other Quiet Revolution: National Identities in English Canada, 1945-1971. Vancouver : University of British Columbia Press, 2005.

Lacombe, Sylvie. La rencontre de deux peuples élus : comparaison des ambitions nationale et impériale au Canada en 1896 et 1920. Sainte-Foy : Presses de l'Université Laval, 2002. 
Lamonde, Yvan. Ni avec eux ni sans eux : le Québec et les États-Unis. Québec : Nuit Blanche, 1996.

Létourneau, Jocelyn. Passer à l'avenir : histoire, mémoire, identité dans le Québec d'aujourd'hui. Montréal : Boréal, 2000.

Lévesque, Andrée. « Réflexions sur l'histoire des femmes dans l'histoire du Québec », Revue d'histoire de l'Amérique française 51.2 (automne 1997).

Louder, Dean R. et Eric Waddell. Du continent perdu à l'archipel retrouvé : le Québec et l'Amérique française. Québec : Presses de l'Université Laval, 2007.

Martel, Marcel. Le deuil d'un pays imaginé : rêves, luttes et déroute du Canada français : les rapports entre le Québec et la francophonie canadienne (18671975). Ottawa : Presses de l'Université d'Ottawa, 1997.

McKay, Ian. "The Liberal Order Framework: A Prospectus for a Reconnaissance of Canadian History", The Canadian Historical Review 81.4 (décembre 2000).

Moon, Bruce. "Paradigms in Migration Research", Progress in Human Geography 19.4 (décembre 1995).

Palmer, Howard. "Mosaic versus Melting Pot? Immigration and Ethnicity in Canada and the United States", International Journal 31.3 (1976).

Perry, Adele. On the Edge of Empire: Gender, Race, and the Making of British Columbia, 1849-1871. Toronto : University of Toronto Press, 2001.

Ramirez, Bruno. "Borderland Studies and Migration: The Canada/United States Case", Repositioning North American Migration History: New Directions in Modern Continental Migration, Citizenship, and Community. Éd. Marc S. Rodriguez, Rochester : University Rochester Press, 2004.

Ramirez, Bruno. "On the Move: French-Canadian and Italian Migrants in the North Atlantic Economy, 1860-1914”, The Canadian Historical Review 74 (décembre 1993).

Ramirez, Bruno. "Shifting Perspectives from the North: Quebec", The Journal of American History 79.2 (septembre 1992).

Roby, Yves. « Les Canadiens français des États-Unis (1860-1900) : dévoyés ou missionnaires ", Revue d'histoire de l'Amérique française 41.1 (été 1987).

Rouillard, Jacques. Ah les États! : les travailleurs canadiens-français dans l'industrie textile de la Nouvelle-Angleterre d'après le témoignage des derniers migrants. Montréal : Boréal express, 1985.

Saul, John Ralston. A Fair Country: Telling Truths about Canada. Toronto : Viking Canada, 2008.

Silver, Arthur. The French-Canadian Idea of Confederation, 1864-1900. Toronto : University of Toronto Press, 1997.

Simpson, Jeffrey. Star-Spangled Canadians: Canadians Living the American Dream. Toronto : Harper Collins, 2001.

Thernstrom, Stephan. Poverty and Progress: Social Mobility in the 19th Century. New York : Atheneum, 1971.

Tremblay, Rémy. Floribec. Espace et communauté. Ottawa : Presses de l'Université d'Ottawa, 2006.

Wilton, Carol. Popular Politics and Political Culture in Upper Canada, 1800-1850, Montréal : McGill-Queen's University Press, 2000.

Woods, Robert. "Chapter 4: Migration Theories", Theoretical Population Geography. London : Longman, 1982. 
Widdis, Randy. "Migration, Borderlands, and National Identity", Permeable Border: The Great Lakes Basin as Transnational Region, 1650-1990, Éd. John J. Bukowczyk, Calgary : University of Calgary Press, 2005.

Widdis, Randy. With Scarcely a Ripple: Anglo-Canadian Migration Into the United States and Western Canada, 1880-1920. Montréal : McGill-Queen's University Press, 1999. 